\title{
Serum IgG4 Concentration is a Potential Predictive Biomarker in Glucocorticoid Treatment for Idiopathic Retroperitoneal Fibrosis
}

Shoichiro Mukai ( $\square$ syoichiro_mukai@med.miyazaki-u.ac.jp )

University of Miyazaki

Naotaka Sakamoto

National Hospital Organization Kyushu Medical Center

Hiroaki Kakinoki

Saga University

Tadamasa Shibuya

Oita University

Ryosuke Moriya

University of Occupational and Environmental Health

Kiyoaki Nishihara

Kurume University School of Medicine

Mitsuru Noguchi

Saga University

Toshitaka Shin

Oita University

Naohiro Fujimoto

University of Occupational and Environmental Health

Tsukasa Igawa

Kurume University School of Medicine

Tatsu Ishii

Fukuoka University Chikushi Hospital

Nobuhiro Haga

Fukuoka University

Hideki Enokida

Kagoshima University

Masatoshi Eto

Kyushu University

Tomomi Kamba

Kumamoto University

Hideki Sakai 
Nagasaki University Graduate School of Biomedical Sciences

\section{Seiichi Saito}

University of the Ryukyus

\section{Naoki Terada}

University of Miyazaki

\section{Toshiyuki Kamoto}

\section{University of Miyazaki}

\section{Research Article}

\section{Keywords:}

Posted Date: January 14th, 2022

DOI: https://doi.org/10.21203/rs.3.rs-1206790/v1

License: (c) (i) This work is licensed under a Creative Commons Attribution 4.0 International License. Read Full License 


\section{Abstract}

Objectives: To evaluate the management and outcome of idiopathic retroperitoneal fibrosis (iRPF) in Japan, and identify its clinical biomarker.

Methods: We retrospectively analyzed 129 patients with iRPF treated between January 2008 and May 2018 at 12 university and related hospitals. Patients treated with glucocorticoid were analyzed to identify a predictive biomarker. These patients were classified into three groups according to overall effectiveness (no change: NC, complete response: $\mathrm{CR}$ and partial response groups: PR), and each parameter was compared statistically.

Results: Male-female ratio was 5: 1, and median age at diagnosis was 69 (33-86) years. Smoking history was reported in $59.6 \%$ of the patients. As treatment, 95 patients received glucocorticoid therapy with an overall response rate of $84 \%$. As a result, serum concentration of IgG4 was significantly decreased in NC group compared with the other two groups $(56.6 \mathrm{mg} / \mathrm{dL}$ vs $255 \mathrm{mg} / \mathrm{dL}, 206 \mathrm{mg} / \mathrm{dL}, P=0.0059$ and 0.0078 ). ROC analysis was performed between the non-responder (NC) and responder groups (CR+PR) to identify the cut-off value of serum IgG4 as a predictive marker. As a result, AUC was 0.793 and the values of sensitivity and specificity were 0.85 and 0.64 , respectively, under the cut-off values of $67.6 \mathrm{mg} / \mathrm{dL}$.

Conclusions: In the majority of iRPF patients, glucocorticoid therapy resulted in a favorable response. Pretreatment serum IgG4 concentration may have potential as a predictive biomarker of steroid treatment.

\section{Introduction}

Retroperitoneal fibrosis (RPF) is characterized by the presence of fibrotic tissue in retroperitoneum, accompanying chronic inflammation, fibrotic tissue surrounding the abdominal aorta and iliac arteries, and ureteral obstruction ${ }^{1-5}$. The presence of fibrotic tissue in retroperitoneum is common in all cases, whereas accompanying chronic inflammation, fibrotic tissue surrounding the abdominal aorta and iliac arteries, and ureteral obstruction are observed to varying degrees in individual cases. Because of its rarity and benign characteristics, accepted evidence seems to be lacking. Therefore, epidemiology, classification, and etiology as well as treatment procedures and information on outcome remain elusive, and this has acted as an obstacle to the establishment of guidelines ${ }^{1-5}$. Published reports estimate the incidence of RFP to be $0.1-1.3 / 100,000$ persons per year, a male-to-female ratio of 2:1-3:1, and a mean age at diagnosis ranging between 55 and 60 years $^{3-8}$.

RPF has been classified into secondary and idiopathic type ${ }^{1-5}$. Secondary RPF includes drug-induced and infectious disease-related etiologies. However, there is no clear evidence regarding the relationship between beta-adrenergic blocker or aspirin-intake and RPF except for ergot alkaloids and dopamine agonists 3,9 . In addition, surgery or radiation therapy for retroperitoneal lesion also induce fibrotic change which may cause secondary $\mathrm{RPF}^{1-5}$. Malignant retroperitoneal neoplasm has been included in the criteria of secondary $\mathrm{RPF}^{1-5}$. On the other hand, $70-80 \%$ of RPF is classified as idiopathic type, which includes 
immunoglobulin G4-related disease (IgG4RD) and IgG4-unrelated chronic aortitis, inflammatory aortic aneurysm, and perianeurysmal fibrosis ${ }^{1-6}$. Although glucocorticoid therapy shows a good response rate in idiopathic RPF (iRPF), predictive biomarkers for the therapy haves yet to be established.

Because the rarity of this disease makes it difficult to collect an adequate number of patients at a local institution, we planned collaborative research with twelve universities and eight related hospitals in the Kyushu-Okinawa area to collect data on RPF patients. In the present report, we retrospectively evaluated clinical data including etiology, management and the outcome of the RPF patients, and discussed serum IgG4 concentration as a predictive biomarker in glucocorticoid therapy.

\section{Method}

\section{Patients}

The present multi-institutional study was conducted with the approval of all institutional review boards (approval No. 0-0336, 2018). We retrospectively analyzed patients with RPF treated between January 2008 and May 2018 at 12 university hospitals and related hospitals. Major criteria were presence of fibrotic tissue in retroperitoneal area without regard to the presence or absence of accompanying inflammation or hydronephrosis. No apparent primary retroperitoneal tumor and retroperitoneal metastasis were observed. Malignant neoplasm occurring in locations other than the retroperitoneal area was included in iRPF. Surgical intervention, radiation to the retroperitoneal area and exact drug-induced RPF (ergot alkaloids and dopamine agonists) were defined as secondary RPF (Figure 1). Patients with IgG4-related disease were included in iRPF. Clinical data including age, gender, comorbidity, past history, results of laboratory examination, appearance of imaging examination, urological examination, details of treatment and the outcome were retrospectively extracted from clinical records.

\section{Baseline Measurements}

Baseline laboratory examination, including complete blood cell count, serum level of creatinine, C-reactive protein (CRP), soluble interleukin-2 receptor (sIL-2R), IgG and IgG4, were performed during the pretreatment period. Plaque size was analyzed by computed tomography (CT) and/or magnetic resonance imaging (MRI).

\section{Evaluation Of Treatment Outcome}

Mass reduction rate was calculated using the changing value of maximum diameter. Response of medical treatment was judged as follows: reduction rate of $>15 \%$ for "reduction" (especially, $>90 \%$ as "complete reduction"); 0-15\% as "no reduction". 
Improvement of urinary tract was evaluated by degree of obstruction (hydronephrosis) in upper urinary tract and condition of ureteral stent. Response was classified into the following four groups: "stent free from the beginning"; "became stent free"; "improved but, still stenting"; "no improvement". The first two groups were judged as "improved", and others were no "improvement".

Overall effect of treatment was analyzed both by mass reduction rate and improvement of urinary tract, and then classified into three groups of "complete response: CR", "partial response: PR", and "no change: NC". Patients classified into CR include both complete reduction and stent free, PR contains reduction and/or improved, and NC consists of both no reduction and no improvement.

\section{Statistical analysis}

This is a fact-finding study without control cohort. Therefore, the result is aggregate data with limited statistical analysis. Intergroup differences were analyzed using the Mann-Whitney U-test (StatFlex version 7) to identify a predictive biomarker of glucocorticoid therapy. A P value of less than 0.05 was considered statistically significant. Receiver operating characteristic (ROC) analysis was also performed using StatFlex version 7, and cut-off value was identified by the Youden index.

\section{Result}

During the study period, a total of 145 RPF patients were treated at each hospital, and a total of 129 patients were classified as idiopathic RPF (iRPF). Initial patient characteristics are shown in Table 1. As a result, there were 108 male patients with a male-female ratio of 5.1: 1.0. Median age at diagnosis was 69 (33-86) years. Smoking history was reported in $59.6 \%$ of the patients. Review of comorbidity and past history revealed autoimmune disease in $14.3 \%$, allergy in $8.6 \%$, non-retroperitoneal malignant neoplasm in $13.4 \%$ and arteriosclerotic disease in $32.8 \%$. Abdominal surgery not within the retroperitoneal area was performed in $18.1 \%$ of the patients (transurethral resection of bladder tumor was included in iRPF). One patient received radiation therapy not within the retroperitoneal area. No patients were treated with ergot alkaloids or dopamine agonists. Aspirin was administered in 10 cases, beta-adrenergic blocker in 7 and methotrexate in 1 case. We classified these patients into idiopathic RPF because evidence in past literature suggesting that aspirin, beta-adrenergic blocker or methotrexate are causes of secondary RPF is lacking ${ }^{3,9}$.

The breakdown of treatment administered to the 127 patients (two patients had no information for treatment) was glucocorticoid in 95 cases, Saireito in 6 cases, mizoribine in 1 case, and two agents (glucocorticoid and rituximab) in 1 case. Surgical intervention was performed in 7 cases, and 17 patients received no treatment (only urinary tract management and observation).

We focused on glucocorticoid therapy, which is a major treatment of iRPF. A total of 94 patients with evaluable outcome were analyzed. As a result, reduction of plaque size was observed in $79(84 \%)$ cases at the median observation period of 82 days (interquartile range: $34-120$ days). Of interest is that 
complete reduction was observed in 13 cases treated by glucocorticoid. Treatment effect for urinary tract was analyzed in 89 cases. Improvement was observed in 62 (69.7\%) cases. Among 58 patients with ureteral stent inserted prior to glucocorticoid treatment, 31 (53.4\%) patients became stent free as a result of treatment. The overall response rate in this study was $84 \%(79 / 94)$.

Reduction of plaque size was observed in 6 cases in the non-treatment group; however, details were unclear because of a very short observation period. Surgical intervention was performed in 8 cases; however, ureteral injury occurred in 2 cases, and 5 cases continued to be managed by stenting after surgery.

We then analyzed the parameters of patients that received glucocorticoid therapy to identify a predictive biomarker. As shown in Table 2, patients were classified into three groups according to overall effectiveness, and each parameter was compared statistically. As a result, serum concentration of IgG4 was significantly decreased in the NC group compared with the other two groups (Figure 2a, $P=0.0059$ and 0.0078 , respectively). However, no statistical difference was observed in serum IgG and sIL2-R (Figure 2b-c).

ROC analysis was performed between non-responder $(N C, n=11)$ and responder groups (CR and PR, $n=60)$ to identify the cut-off value of serum IgG4 as a predictive marker. ROC curve, cut-off value and area under the curve (AUC) are shown in Figure 3. As a result, the cut-off value was $67.6 \mathrm{mg} / \mathrm{dL}$, and AUC was 0.793 . The values of sensitivity and specificity were 0.85 and 0.636 .

Contents of glucocorticoid therapy is shown in Table 3. Initial treatment period of responder group, and maintenance treatment period of non-responder group seemed to be higher; however, no significant differences were noted for each group.

\section{Discussion}

In this study, we collaborated with 12 university and 8 related hospitals to collect clinical data on 144 RPF patients. The study was a retrospective investigation aimed at fact-finding; therefore, statistical analysis was problematic due to missing values (data) as well as differences among hospitals in diagnostic and treatment strategies, and evaluation of outcomes. However, recent real RPF treatment data in the KyushuOkinawa area was available, allowing this fact-finding study to include the largest number of Japanese patients to date.

Diagnosis of RPF was based on imaging studies performed by attending physicians in each hospital with or without pathological diagnosis. The primary diagnostic finding for inclusion was presence of fibrotic tissue in retroperitoneal lesion. Presence of ureteral obstruction or inflammation were not required for inclusion. Coexistence of malignancy in different lesions was included. Patient history of radiation and surgical intervention in retroperitoneal area were included as secondary RPF. No patients had apparent systemic infectious disease. According to our criteria, 15 patients were classified as secondary RPF, and 
129 patients were diagnosed with iRPF. However, prompt establishment of exact global diagnostic criteria is strongly recommended.

In our study, mean age at diagnosis was slightly higher than that published in previous reports ${ }^{2-7}$. Although this was not a population-based study, male predominance was apparent with a male-to-female ratio of 5.1: 1.0. The result was higher than that found in the literature ${ }^{2-7}$. As a risk factor, a significant association between RPF and smoking has been reported. In this case-control study, the risk was higher (odds ratio of 3.21) in current smokers and former smokers (odds ratio of 2.93) compared with individuals with no history of smoking ${ }^{10}$. Indeed, a high incidence (59.6\%) of smoking history was observed in our study, and the result is consistent with previous reports. RPF has been reported to include chronic periaortitis, perianeurysmal fibrosis and inflammatory abdominal aortic aneurysm, which suggests a meaningful correlation between arteriosclerotic disease and $\mathrm{RPF}^{2-6}$. Compared with previous studies, the incidence of arteriosclerotic disease as a comorbidity was high (32.8\%), and the incidence of autoimmune disease was similar (14.3\%) in the current study ${ }^{2-6}$. In addition, results of 17 patients administered aspirin and beta-adrenergic blocker may correlate with arteriosclerotic disease.

Outcome of iRPF in reduction of size and/or urinary tract improvement was evaluated for a total of 126 patients in this study. As a result, glucocorticoid therapy achieved a favorable response rate (84\%), and ureteral stent was removed in $53.4 \%$ of the patients. The response rate was slightly higher than previous reports $^{2-6}$. The results indicate the apparent efficacy of glucocorticoid, and that glucocorticoid therapy is a standard first-line treatment for iRPF. As the next step, standardization of treatment protocol is necessary. In the literature, the recommended treatment strategy is an initial dose of $0.6-1 \mathrm{mg} / \mathrm{kg} / \mathrm{day}$ of prednisolone for 2-4 weeks, with the dosage gradually tapered to $2.5-5 \mathrm{mg} /$ day over a period of greater than 6 months ${ }^{2}$. Tanaka et al. suggested the convenience of treatment initiated with $0.6 \mathrm{mg} / \mathrm{kg} / \mathrm{day}$ of prednisolone for 4weeks, and then a 10\% reduction in dosage every 2 weeks for IgG4-related disease $(\operatorname{IgG} 4 \mathrm{RD})^{5}$. Indeed, median initial dose was $30 \mathrm{mg}$ for 26.5 days in the current study, and a total of $46 \%$ of the patients started with $30 \mathrm{mg} /$ day of prednisolone. The results of our study were similar to the protocol for IgG4RD, and the efficacy seemed to be acceptable. No significant differences were noted between responder group and non-responder group in treatment dose and period in both initial and maintenance phase. However, the maintenance treatment period in non-responder group seemed to be longer than in responder group, which suggests unnecessarily prolonged treatment. A prospective study analyzing both efficacy and safety would be helpful in establishing a standard protocol for iRPF.

Recently, the majority of idiopathic RPF has been classified as IgG4RD, and greater than half of idiopathic RPF patients were reported to have been diagnosed with IgG4RD by histological examination ${ }^{4}$. However, the exact pathophysiology is controversial due to the fact that fundamental IgG4RD is a systemic disease, whereas RPF occurs in a limited area of the retroperitoneum. Frequency of concurrent RPF in IgG4RD has been reported as $3-19 \%{ }^{11}$, and approximately $60 \%$ of RPF is reported to be associated with IgG4RD ${ }^{12}$. However, no systematic analysis of a large cohort to evaluate the association with IgG4RD has been conducted. In the current study, only 7 cases were diagnosed as IgG4RD by pathological analysis; 
however, a total of 69 patients were analyzed for serum IgG4 concentration, and elevation (cut-off value of $<125 \mathrm{mg} / \mathrm{dL}$ ) was observed in 43 patients $(62.3 \%)$. Interestingly, patients with high serum IgG4 concentration showed a favorable response to glucocorticoid therapy. In spite of the limitation of our study, statistical significance was observed. Although the exact number of potentially overlapped IgG4RD could not be evaluated, serum IgG4 concentration may be a candidate as a predictive biomarker of glucocorticoid therapy. In this study, acceptable AUC (0.793) was observed, and cut-off value was calculated as $67.6 \mathrm{mg} / \mathrm{dL}$. However, the values of sensitivity and specificity ( 0.85 and 0.636$)$ might not be sufficient as cut-off levels. Further prospective examination is recommended to clarify our result.

A tendency of high serum IgG and low sIL-2R concentration was also observed in the favorable response group; however, no statistical significance was observed (Figure 2b-c). Serum sIL2-R was examined to rule out malignant lymphoma. As a result, increased serum level was observed in $74.5 \%$; however, the degree of increase was not significant compared with that of malignant lymphoma ${ }^{13}$. RPF is also associated with inflammation. In addition, infiltration of both B cells (and/or IgG4-positive plasma cell) and $T$ cells has also been confirmed pathologically. Therefore, the phenomenon may not be a diseasespecific result; however, our investigation revealed that this is the first time that elevated serum sIL2-R level has been described in RPF.

As mentioned above, limitations of our study are its being a retrospective investigation, which created obstacles to accurate statistical analysis due to missing values (data), and differences among hospitals in diagnostic and treatment strategies as well as evaluation of outcomes.

\section{Conclusion}

We conducted a fact-finding study on 129 iRPF patients treated in the Kyushu-Okinawa area of Japan. The majority of iRPF patients received glucocorticoid therapy and achieved a favorable response. As a predictive biomarker of steroid treatment, pre-treatment serum IgG4 concentration may be useful. The establishment of acceptable guidelines is strongly recommended.

\section{Abbreviations}

RPF

Retroperitoneal fibrosis

iRPF

Idiopathic retroperitoneal fibrosis

CRP

C-reactive protein

sIL2-R

soluble interleukin-2 receptor

$\lg G$

Immunoglobulin G 
$\lg$ G4RD

Immunoglobulin G4-related disease

ROC

Receiver operating characteristic

\section{Declarations}

\section{Acknowledgment}

The authors would like to thanks urology department of Kyushu central hospital of the mutual aid association of public school teachers, Oita prefectural hospital, Kagoshima city hospital, Miyazaki prefectural hospital, Harasanshin hospital, National hospital organization Kyushu cancer center and Chubu Tokushukai hospital for data collection. We also thank Dr. Hiromasa Tsukino of urology department of Junwakai memorial hospital, and Ms Miyuki Akino of urology department of Miyazaki university for support of statistical analysis.

\section{Funding}

No funding was provided for this study.

\section{Author's Contributions}

All authors participated in creating the study design. SM analyzed all data and wrote manuscript. SM, NT and TK designed the study. NS, HK, TS, RM, KN, MN acquired data and organized. TS, NF, TI, TI, NH, HE, $\mathrm{ME}, \mathrm{TK}, \mathrm{HS}$, SS provided a critical revision of this study. All the authors have read and approved the final manuscript.

\section{Availability of data and materials}

The datasets generated and/or analyzed during the current study are not publicly available due to identifiable patient information but are available from the corresponding author on reasonable request.

\section{Ethics approval and consent to participate}

The study was conducted in accordance with the Declaration of Helsinki (as revised in 2013). This study was approved by Ethics committee of Miyazaki University (Approval No. 0-0336, 2018). All participants provided written informed consent.

\section{Consent for publication}

Not applicate.

\section{Conflict of interest: None declared.}

The authors declare that they have no competing interests. 


\section{References}

1. Vaglio A, Salvarani C, Buzio C. Retroperitoneal fibrosis. Lancet. 2006; 21: 367(9506):241-251

2. Vaglio A, Maritati F.J. Idiopathic Retroperitoneal Fibrosis. Am Soc Nephrol. 2016; 27(7): 1880-1889.

3. Urban ML, Palmisano A, Nicastro M, Corradi D, Buzip C, Vaglio A. Idiopathic and secondary forms of retroperitoneal fibrosis: a diagnostic approach. Rev Med Interne. 2015; 36(1): 15-21.

4. Rossi GM, Rocco R, Accorsi Buttini E, Marvisi C, Vaglio A. Idiopathic retroperitoneal fibrosis and its overlap with IgG4-related disease.

5. Intern Emerg Med. 2017 Apr;12(3):287-299.

6. Tanaka T, Masumori N. Current approach to diagnosis and management of retroperitoneal fibrosis. Int J Urol. 2020; 27(5): 387-394.

7. Kermani TA, Crowson CS, Achenbach SJ, Luthra HS. Idiopathic retroperitoneal fibrosis: a retrospective review of clinical presentation, treatment, and outcomes. Mayo Clin Proc. 2011; 86(4): 297-303.

8. Zhou HJ, Yan Y, Zhou B, Lan TF, Wang XY, Li CS. Retroperitoneal fibrosis: a retrospective clinical data analysis of 30 patients in a 10-year period. Chin Med J (Engl). 2015; 128(6): 804-810.

9. Scheel PJ Jr, Feeley N. Retroperitoneal fibrosis: the clinical, laboratory, and radiographic presentation. Medicine (Baltimore). 2009; 88(4):202-207.

10. Pryor JP, Castle WM, Dukes DC, Smith JC, Watson ME, Williams JL. Do beta-adrenoceptor blocking drugs cause retroperitoneal fibrosis? Br Med J (Clin Res Ed). 1983; 287(6393): 639-641.

11. Goldoni M, Bonini S, Urban ML, Palmisano A, De Palma G, Galletti E, Coggiola M, Buzio C, Mutti A, Vaglio A. Asbestos and smoking as risk factors for idiopathic retroperitoneal fibrosis: a case-control study. Ann Intern Med. 2014; 161(3):181-188.

12. Hara N, Kawaguchi M, Takeda K, Zen Y. Retroperitoneal disorders associated with IgG4-related autoimmune pancreatitis. World J Gastroenterol. 2014; 20(44): 16550-16558.

13. Fujimori $\mathrm{N}$, Ito $\mathrm{T}$, Igarashi $\mathrm{H}$, Oono T, Nakamura T, Niina Y, Hijioka M, Lee L, Uchida M, Takayanagi R. Retroperitoneal fibrosis associated with immunoglobulin G4-related disease. World J Gastroenterol. 2013; 19(1): 35-41.

14. Murakami J, Arita K, Wada A, Mihara H, Origasa H, Kigawa M, Yasuda I, Sato T. Serum soluble interleukin-2 receptor levels for screening for malignant lymphomas and differential diagnosis from other conditions. Mol Clin Oncol. 2019;11(5): 474-482.

\section{Tables}

Due to technical limitations, tables 1 to 3 are only available as a download in the Supplemental Files section.

\section{Figures}


Figure 1

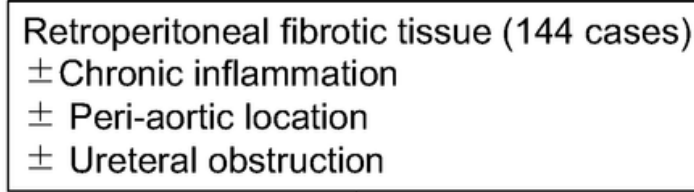

Secondary RPF (15 cases)

Drugs: ergot alkaloids, dopamine agonists ( 0 case)

Surgery: retroperitoneal area (12 cases)

Radiation: retroperitoneal area (3 cases)

Primary or metastatic tumor of retroperitoneum (0 case)

Idiopathic RPF (129 cases)

\section{Figure 1}

Classification of retroperitoneal fibrosis, and selection of study cohort. A total of 144 cases were enrolled, and 15 cases were classified as secondary RPF. Patients with idiopathic RPF were analyzed in this study. 
(a)

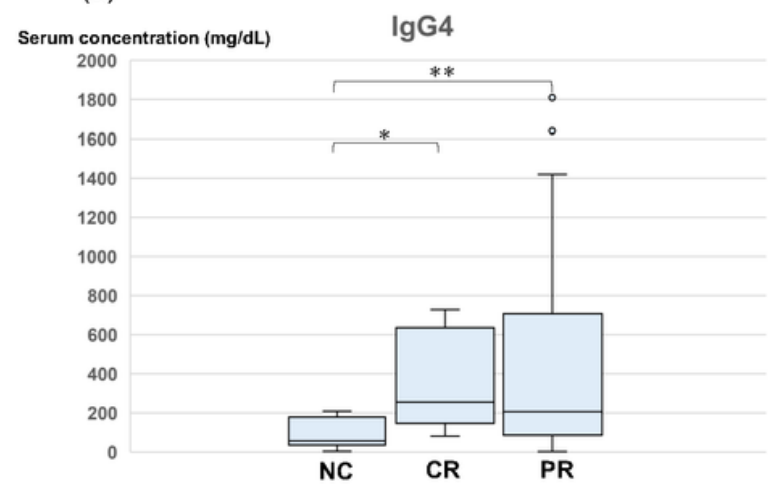

Figure 2 (b)

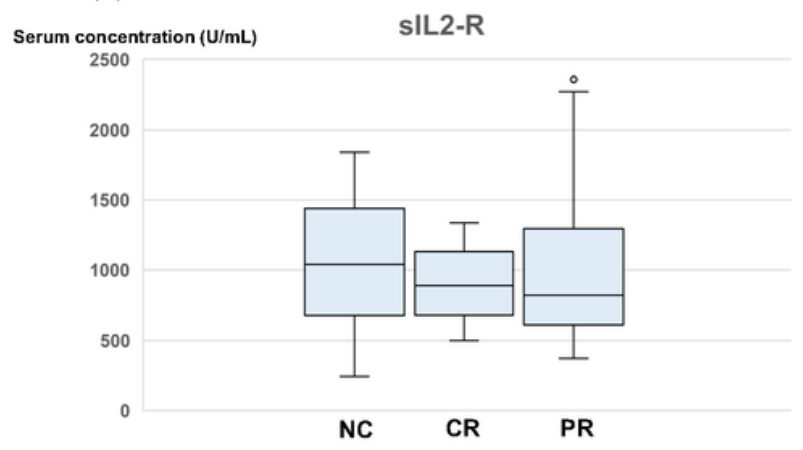

(c)

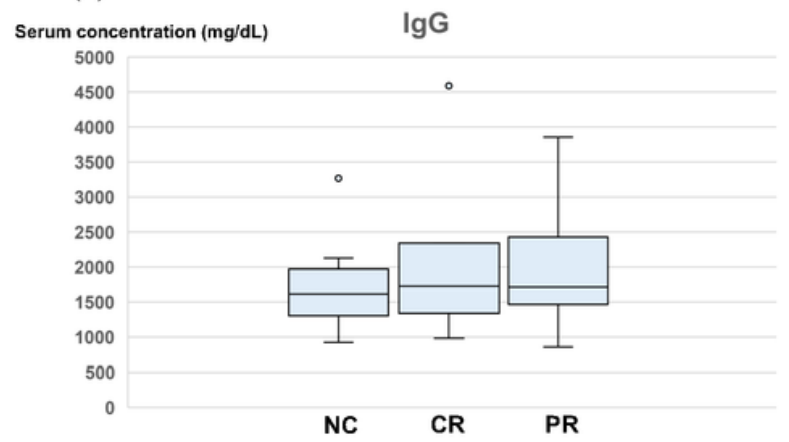

\section{Figure 2}

Serum concentration of IgG4 (a), sIL2-R (b) and IgG (c) in each group classified by response to glucocorticoid therapy. Intergroup differences were analyzed using the Mann-Whitney $U$-test. A P value $<0.05$ was considered statistically significant.

IgG4, Immunoglobulin G4; sIL2-R, soluble interleukin-2 receptor; NC, no change; CR, complete response; PR, partial response.

*P=0.0059; ** $P=0.0078$. 
Figure 3

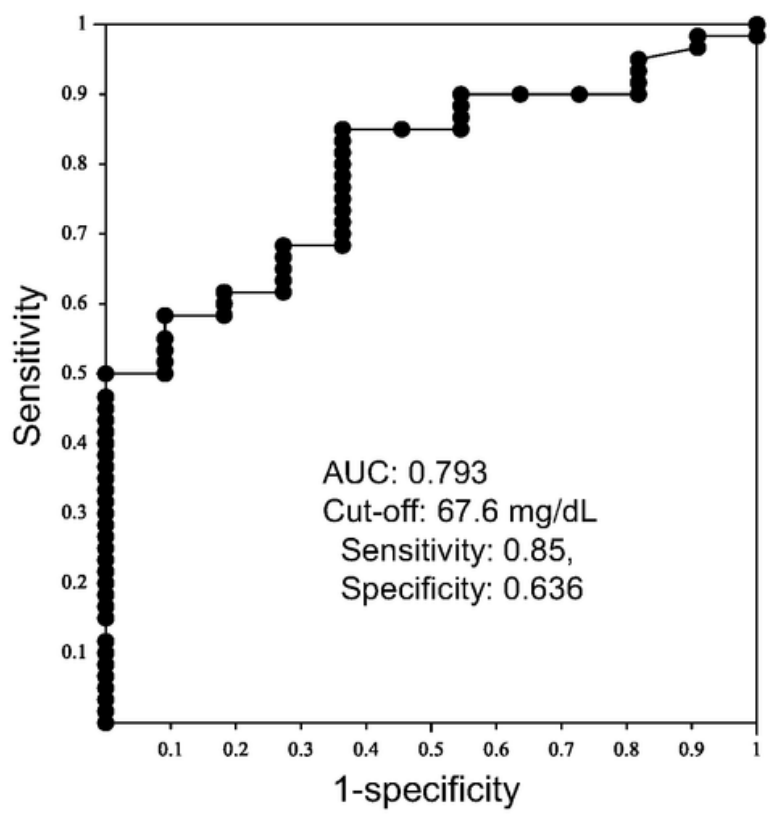

\section{Figure 3}

Receiver operating characteristic (ROC) curve analysis of IgG4levels.

The area under the ROC curve and cut-off value were calculated using responder group ( $\mathrm{n}=60)$ and nonresponder group $(\mathrm{n}=11)$ data.

$A U C$, area under the curve

\section{Supplementary Files}

This is a list of supplementary files associated with this preprint. Click to download.

- Table1.pdf

- Table2.pdf

- Table3.pdf 